\title{
Revisión epistemológica de nuevas tipologías urbanas para la identificación de mini ciudades en América Central
}

\author{
Sabrine Acosta Schnell
}

Invitada internacional

Instituto de Altos Estudios de América Latina, Université Sorbonne Nouvelle Paris 3, Francia.

sabrigeografia@hotmail.com

Recibido: 15 de Mayo del 2017

Aprobado: 4 de Agosto del 2017

\section{Sabrine Acosta Schnel}

Bachillerato en Geografía en la Universidad de Costa Rica. Máster en Geografía con énfasis en redes, ordenamiento territorial y políticas públicas en la Universidad Federal de Santa Catarina, Brasil. Cursa el doctorado en Geografía con énfasis en ordenamiento territorial en el Instituto de Altos Estudios de América Latina, Université Sorbonne Nouvelle Paris 3, Francia. Áreas de interés: mercado inmobiliario, ordenamiento territorial, el proceso de verticalización en Centroamérica, las mini ciudades y la geografía histórica.

\begin{abstract}
Resumen:
Las mini ciudades en Centroamérica son nuevas tipologías urbanas que se pueden identificar como proyectos de uso mixto con espacios residenciales, comerciales y de entretenimiento, de iniciativa privada. La novedad de esta tipología urbana sugiere un acercamiento desde la geografía urbana ya que no se han investigado previamente. Este es el objetivo principal de esta revisión epistemológica sobre los abordajes de tipologías urbanas como barrios cerrados, centros comerciales y parques temáticos para intentar caracterizar las mini ciudades centroamericanas. Esta revisión bibliográfica hace parte de una tesis doctoral que aborda la temática específicamente para el caso costarricense y guatemalteco. La revisión bibliográfica no pretendió ser exhaustiva ni profunda sino crítica y con carácter de diagnóstico. Se señalan seis líneas temáticas que incluyen: i) las discusiones sobre el riesgo de simplificar la teoría de las tipologías urbanas a todo el continente americano; ii) los análisis de las ciudades con un amplio recorte histórico, desde la antigüedad hasta las actuales tendencias; iii) las búsquedas de la definición de ciudad; iv) las investigaciones sobre los centros comerciales y parques temáticos que emulan ser mini ciudades; v) abordajes de la reorganización del espacio urbano y su constante carácter dinámico; vi) los faltantes (gaps) en la teoría de la geografía urbana centroamericana, específicamente sobre las recientes mini ciudades. Se concluye reflexionando sobre la necesidad de aportar un análisis específico sobre las mini ciudades como nuevas tipologías urbanas en Centroamérica.
\end{abstract}

Palabras clave: barrios cerrados; centros comerciales; parques temáticos; mini ciudades

\section{Epistemologic review of new urban typologies for the identification of mini cities in Central America}

\begin{abstract}
:
Central American mini cities are new urban typologies that can be identified as mixed-use projects with residential, commercial and entertainment spaces of private initiative. The novelty of this urban typology suggests an approach from the urban geography since it has not been previously investigated. This is the main objective of this epistemological review of the approaches of urban typologies like gated communities, commercial centres and "Theme parks" to try to characterize the Central American mini cities. This bibliographic review is part of a doctoral thesis that addresses the issue specifically for the Costa Rican and Guatemalan cases. The bibliographic review was not intended to be exhaustive or thorough, but critical and diagnostic. Six main themes are identified. These include discussions on the risk of simplifying the theory of urban typologies to the entire American continent. Also, several productions used a wide historical cut from the antiquity to the current trends. Another line is the search for the definition of city to adapt it to the current proposals of mini cities. There is also research on malls and theme parks that emulate mini cities. Another recurring theme is the constant reorganization of urban space and its constant dynamic character. Finally, gaps were identified in the theory of urban geography in Central America, specifically on the recent mini-cities. It is concluded by reflecting on the need to provide a specific analysis on mini cities as new urban typologies in Central America.
\end{abstract}

Keywords: gated communities; shopping centre; themed parks; mini cities. 


\section{Introducción}

as mini ciudades centroamericanas son una nueva tipología urbana que reúne características de barrios cerrados, centros comerciales y parques temáticos. Se podría proponer que se clasifiquen como una cuarta tipología a parte de las ya mencionadas ya que lo que las diferencia de las demás es justamente su carácter de uso mixto. En este contexto, la presente revisión epistemológica inicialmente propuso la búsqueda bibliográfica contemplando aportes científicos, específicamente, sobre mini ciudades. No obstante, debido al carácter novedoso de estos recientes productos inmobiliarios, no se encontró producción específica al respecto. En este contexto, la metodología se replanteó y el presente artículo propone una revisión epistemológica crítica sobre la producción científica, en el continente americano, de las tipologías que contribuyen a caracterizar las mini ciudades (barrios cerrados, centros comerciales y parques temáticos).

El trabajo es parte de una tesis doctoral de mi autoría que analiza las mini ciudades como nuevas tipologías urbanas, especificamente en Costa Rica y Guatemala1. Centroamérica es una región que se ha diferenciado marcadamente en su desarrollo histórico geográfico con respecto al resto de América Latina y sus tendencias inmobiliarias presentan características propias poco analizadas en la literatura desde la geografía urbana.

Cabe resaltar que las mini ciudades no son simplemente barrios cerrados. Las mini ciudades son caracterizadas por proyectos de uso mixto donde se combinan espacios de recreo, residencias y comercio. Los barrios cerrados, por el contrario, son proyectos monofuncionales que han caracterizado la expansión urbana horizontal local desde los últimos treinta años, aproximadamente. Las mini ciudades, por su parte, se han observado desde la última década y actualmente presentan un auge especialmente en Costa Rica.

Para aclarar los alcances del presente trabajo, se resalta que la revisión no pretende ser exhaustiva ni profunda sino más bien de carácter crítico resaltando el carácter de diagnóstico de ciertas obras seleccionadas. Se conoce que los aportes científicos son vastos sobre las tipologías urbanas mencionadas y no se pretende redundar en discusiones ya planteadas. Este trabajo tampoco procura ahondar específicamente en la discusión sobre las mini ciudades sino en los textos científicos que podrían aportar a su discusión, motivo por el cual la discusión propiamente de las mini ciudades se colocará en segundo plano.

Se amplió la búsqueda de los aportes científicos de la región centroamericana para abarcar una revisión literaria de todo el continente americano. Si bien es cierto que el caso centroamericano cuenta con diversa producción en la temática de barrios cerrados, estas mini ciudades son una tendencia que ha caracterizado el reciente auge inmobiliario en la segunda década del siglo XXI y no se registró ningún aporte científico sobre el tema específico. De esta forma, se justifica la relevancia de la presente revisión bibliográfica.

A nivel latinoamericano se observa una mayor producción científica con un enfoque de análisis individual de cada tipología urbana. Estas se comentan desde una sola perspectiva: analizadas solamente como barrios cerrados o meramente como centros comerciales. $\mathrm{O}$ una u otra. El aporte de la presente investigación es un diagnóstico sobre las discusiones que incluyan las tres tipologías mencionadas como metodología comparativa para el análisis de las mini ciudades como una novedosa tipología híbrida. Esta metodología y esta revisión epistemológica, no han sido registradas previamente en los aportes de la geografía urbana centroamericana.

Título de la Tésis: L'émergence de nouvelles formes de métropolisation en Amérique Centrale: les "néo-villes" à San José (Costa Rica) et à Ciudad de Guatemala (Guatemala). Tésis en preparación en Sorbonne Paris Cité, en el cuadro de la École doctorale Europe latine et Amérique latine (Paris) y de la Université de la Sorbonne Nouvelle (Paris) desde el 20-11-2015. http://www. theses.fr/s156993.
El amplio recorte espacial y temporal obligó a limitar el tamaño de la presente búsqueda, dejando por fuera una amplia gama de aportes científicos, los cuales, en realidad, no difieren mayormente de los seleccionados.

Continuando con la metodología, se realizó la búsqueda en bibliotecas digitales de revistas cientificas (Jstor, ResearchGate) y en libros impresos. Se cubrieron las áreas de arquitectura, urbanismo, geografía, economía y ordenamiento territorial. Diversas revistas inmobiliarias y periódicos (Inversión Inmobiliaria, Inmobilia.com, entre otras) se 
revisaron y evidenciaron críticas a las mini ciudades, sin embargo no encajaban como aportes científicos para el presente ensayo.

Teniendo claro lo previamente explicado, se puede mencionar que el objetivo principal de la actual revisión epistemológica es diagnosticar, de forma crítica, los abordajes de las tipologías urbanas que contribuyen a caracterizar las mini ciudades (barrios cerrados, centros comerciales y parques temáticos).

El ensayo señala cuáles son los conflictos, los patrones temáticos, las faltantes en las discusiones y las tendencias de investigación, para así poder aportar un contexto adaptado a la realidad centroamericana, sin caer en la generalización. También se resaltan puntos de discusión que resultan problemáticos o que corresponden a temas recurrentes, para así también poder señalar las faltantes.

\section{Análisis global de las tendencias}

La presente revisión bibliográfica mostró que durante las últimas décadas predomina un análisis cualitativo con amplios debates sobre temática recurrente. Los debates con contenido cuantitativo aportan novedad sobre casos de estudio locales y esto se da con mayor frecuencia en países con bases de datos más robustas como en el caso estadounidense o el luso americano. En la literatura revisada, abundan las discusiones sobre barrios cerrados y no muestran mayor novedad relacionada a recientes tendencias de los mercados inmobiliarios o la planificación urbana reciente. La tendencia predominante son las conclusiones alrededor de la misma temática sobre la segregación socio espacial y la fragmentación desde un punto de vista negativo.

Resultan interesantes aquellas conclusiones o análisis que logran hacer una correlación entre la teoría general y la especificidad del lugar, como es el caso del geógrafo Michael Janoschka, el cual constantemente alude a las mini ciudades argentinas para amarrar la teoría discutida. Este autor ha realizado importantes aportes en el análisis de enclaves urbanos desde inicio de la década del 2000 en Suramérica. Hay casos de literatura donde se presentan datos cuantitativos como información censal, fechas históricas o marcadores económicos como, por ejemplo, Klin et al. (1991) en Postsuburban California, the transformation of Orange County since World War II, libro que compila obras de investigadores que trabajan en la Universidad de California, principalmente. Este patrón se observa con mayor frecuencia en investigaciones estadounidenses 0 brasileñas, ya que en los casos latinoamericanos predomina un análisis más cualitativo. En el caso de los datos cuantitativos, estos permiten enfatizar la relevancia y veracidad de los argumentos a la hora de afirmar o refutar sus hipótesis. En la obra The evolving spatial form of cities in a globalising world economy, Johannesburg and Sao Paulo (2004), del filósofo y sociólogo Martin J. Murray, él ampara sus discusiones con datos numéricos sobre las realidades del desorden urbano en los casos de estudio.

En general, poco material llega a exponer la metodología utilizada para la recolección de datos. Desde California, el libro Fortress America, Gated Communities in the United States, (Blackely et Snyder, 1997) es uno de los que exponen la metodología utilizada para el análisis de los barrios cerrados. Peter Gotsch (2009), quien hizo su disertación de PhD en arquitectura en Zurich sobre el tema de NeoTowns, también presenta una amplia explicación de la metodología utilizada para identificar estas tipologías. Gotsch (2009) detalladamente estructura su tesis para abarcar diversas perspectivas como la económica, social, histórica y cultural para contextualizar sus tres NeoTowns analizadas: (Bumi Serpong Damai (Jakarta), Navi Mumbai (Mumbai) and Alphaville-Tamboré (São Paulo). El autor logra un balance entre el análisis cuantitativo y cualitativo a lo largo de los capítulos. Describe su nueva tipología como una combinación de New Towns desarrolladas por iniciativa privada en el marco de una nueva tendencia de urbanización contemporánea neoliberal. De ahí el término town con el prefijo neo.

Se decidió incluir casos de estudio y autores fuera del recorte de América Latina, como por ejemplo, del teorista espacial neoyorquino Edward Soja quien expone categorías de análisis que podrían ser revisadas y adaptadas a la realidad local latinoamericana y centroamericana. Este examina el caso del Condado Orange y cómo se crean 
"enclosures for the absolutly fantastic reproduction of the totally real" (Soja, 1992).

Estas ideas pueden ser un punto de partida para identificar patrones semejantes a la creación de mini ciudades o proyectos de uso mixto. Janoschka es otro autor que recurre a los aportes de la literatura estadounidense y demuestra que pueden ser utilizados para enriquecer nuevas características y teorías sobre casos menos estudiados 0 actualizados en Latinoamérica.

Entre las temáticas predominantes identificadas en la literatura revisada, la aceptación general tiende a ser principalmente sobre problemas que acarrean estas formas urbanas. En los debates, es poco frecuente encontrar un material de análisis que rescate puntos positivos o al menos imparciales de la realidad de los barrios cerrados, mini ciudades, centros comerciales o parques temáticos. La mayoría de las lecturas presenta una visión crítica negativa hacia la realidad de los barrios cerrados y centros comerciales; sin embargo Janoschka, a principio de siglo $(2003,2005)$, mantiene en su análisis un matiz más descriptivo para presentar la realidad sudamericana de la manera más objetiva posible. Salcedo y de Simone (2012) publicaron El Mall en Chile 30 años, la cual es una obra bastante completa con carácter de diagnóstico sobre casos de estudios concretos y enriquecida con datos cuantitativos sobre los centros comerciales.

Otras temáticas que predominan son la creciente inseguridad urbana y la segregación social como principales motivos y resultados de la proliferación de estas nuevas formas urbanas en las últimas décadas. Murray (2004), investigador estadounidense, ejemplifica claramente esta teoría en sus casos de estudio de São Paulo y Ciudad del Cabo. Por su parte, Blackely y Snyder realizan sus aportes, para el caso californiano, argumentando que a pesar de que los métodos de barrera pueden brindar la sensación de seguridad, no necesariamente van a traer seguridad a los habitantes. Janoschka (2005) propone otras teorías para explicar la decisión de los habitantes para encapsularse en sus propiedades. En este contexto, la socióloga argentina Maristella Svampa (2002) se enfoca más en la discusión de cómo estas burbujas urbanas afectan las relaciones sociales.

Se resalta, en general, que el carácter comparativo de los estudios resulta más enriquecedor al poder obtener similitudes y diferencias desde diversas perspectivas para poder subrayar la especificidad de los lugares. Larry Ford (2000), geógrafo estadounidense, demuestra que el carácter comparativo entre los casos de estudio es valioso para entender la interacción entre los diferentes valores culturales y la creación del espacio urbano. Capron (1996 y 2004), geógrafa y profesora de sociología en México, lo demuestra en sus comparaciones entre el continente americano y europeo y Gotsch (2009) lo aborda en casos de Asia y Suramérica.

\section{Presentación de las seis temáticas identificadas}

El material revisado se clasificó siguiendo ciertas temáticas identificadas que se repetían en las obras. Los trabajos presentan una o más de estas líneas que sugiero agrupar de la siguiente forma:

1. El modelo de ciudad latinoamericana y el riesgo de sobre simplificar la teoría urbana;

2. el análisis de ciudades con un amplio recorte histórico, desde la antigüedad hasta las actuales tendencias;

3. la constante búsqueda de la definición de ciudad;

4. los centros comerciales y parques temáticos que emulan ser ciudades;

5. la constante reorganización del espacio urbano y su carácter dinámico;

6. los faltantes (gaps) en la teoría de la geografía urbana centroamericana.

Estas seis temáticas son denominadores comunes o acercamientos que la mayoría de los autores utilizan para abordar las tipologías urbanas en cuestión. Si a primera vista parecen no tener relación con las mini ciudades, son abordajes epistemológicos que contribuyen a la identificación y caracterización de estas. 


\section{Comparación y discusión de las seis líneas de análisis}

\section{I) El riesgo de sobre simplificar la teoría urbana}

Los puntos convergentes en estas investigaciones abordan principalmente casos sudamericanos. El caso de Buenos Aires, de São Paulo y de Santiago, son grandes ciudades que concentran diversas obras sobre la temática de los barrios cerrados en el marco del urbanismo neoliberal y destacan los aportes de la argentina Svampa. Sin embargo, se trata de evitar extrapolar los hallazgos en las investigaciones conducidas en estas grandes ciudades y aplicarlos a otras regiones.

El arquitecto e historiador argentino, Adrián Gorelik (2005), plantea que sería hasta "absurdo" intentar definir la ciudad latinoamericana por medio de un ideal de representación de un conjunto de características como si se tratase de un "Frankenstein urbano". Concordando con este autor, los hallazgos sobre "la ciudad latinoamericana", los patrones sobre los barrios cerrados y las grandes mini ciudades bonaerenses como Nordelta, no se pueden sencillamente extrapolar como una tendencia regional. El caso centroamericano tiene sus particularidades. Ignorar la especificidad del desarrollo histórico geográfico de cada región, país o ciudad, sería caer en el error de tratar toda la región latinoamericana como "un laboratorio estéril" donde se desconocería aquello que le da un sentido específico a cada caso.

Por su parte, Gotsch (2009), enfatiza la necesidad de ampliar los estudios sobre las NeoTowns para enriquecer el novedoso debate sobre estas nuevas tipologías urbanas y subrayar especificidad del lugar. El concepto de NeoTowns se construye a partir de diversos escenarios. No es un concepto americano sino que más bien alude a las New Towns de origen inglés inspiradas en la Garden City de Ebenezer Howard, a finales del siglo XIX. Si bien es cierto estas últimas se contextualizan en el marco de la reconstrucción post Primera Guerra Mundial, Gotsch (2009) modifica el término a NeoTowns para actualizarlo a las tendencias contemporáneas de urbanización neoliberal en países no industrializados o economías emergentes.

Continuando con el análisis sobre la especificidad del lugar, Capron (2004) realiza un estudio comparativo de los barrios cerrados en Estados Unidos y Francia, lo cual resulta un aporte novedoso al incluir países cuyos desarrollos histórico-geográficos fueron muy diferentes entre sí para resaltar sus particularidades individuales. Para enfatizar esto, la autora propone metodológicamente utilizar la terminología en el mismo idioma original para evitar distorsionar el campo semántico que alude a las dinámicas socio-espaciales locales, propias de dicho caso de estudio. Por ejemplo, la community es un término con el cual se identifican los estadounidenses para referirse al proceso de compartir un espacio público, valores e ideales. Otros términos aluden más al aspecto jurídico como el condominio, el cual es una categoría de análisis que hace referencia a un tipo de tenencia de la tierra. La variedad del vocabulario permite una visión más profunda de la realidad local y ayuda a evitar generalizaciones estériles.

Murray (2004), por su parte, también indica que acudir a las comparaciones permitiría tener nuevas visiones y conocimientos sobre las dinámicas cambiantes de las ciudades en la economía contemporánea mundial. Los estudios comparativos, como una metodología, suman a la riqueza del entendimiento de la realidad actual ya que la temática de la ciudad y del mercado inmobiliario involucra una red de actores que interactúan a diferentes escalas locales, regionales y mundiales.

Janoschka, es uno de los autores que realiza sus conclusiones tratando de evitar extrapolar interpretaciones. Él va más allá del mainstream sobre los barrios cerrados y propone que uno de los posibles factores para la auto segregación es el deseo de volver a un mundo "más restringido y ordenado" que se asocia al pasado sin caer en la eterna discusión sobre la dicotomía entre seguridad-inseguridad. Resulta innovador este aporte ya que enriquece el debate general que parece haberse estancado en el análisis de la teoría sobre la inseguridad, que incluso predomina en varias producciones centroamericanas. 


\section{II) Un amplio recorte histórico}

Otro patrón de análisis encontrado en la literatura revisada es la utilización de un amplio recorte histórico para retomar puntos de comparación y evolución en la definición e identificación de tipologías urbanas. En el caso de los conjuntos residenciales cerrados, estos se perciben como nuevas tendencias; no obstante estos tienen amplios precedentes históricos (Capron, 2004). Muchas investigaciones retoman detalles desde la antigüedad, la edad media, el periodo colonial, hasta el surgimiento de la línea neoliberal en el siglo XX y las actuales tendencias del Nuevo Urbanismo². Por ejemplo, el geógrafo austriaco, Axel Borsdorf (2003), realiza una amplia cronología sobre las diferentes estructuras cerradas y el papel de los muros en la planificación urbana en general. Propone diversas explicaciones de índole política, económica y social para visualizar cómo los actuales barrios cerrados son resultado de toda una tradición de amplio recorte temporal. Este aporte resulta valioso como metodología para poder explicar diversas características que presentan las actuales mini ciudades centroamericanas, como por ejemplo, su arquitectura, las estrategias de mercadeo, el público meta, sus funciones y hasta su organización interna. Con estas comparaciones se puede esclarecer ese anhelo por el retorno a una imagen de ciudad idílica del pasado.

Gotsch (2009) recomienda fuertemente realizar referencias históricas en el desarrollo de los estudios de las NeoTowns para, por ejemplo, poder entender cómo las mother cities de las NeoTowns analizadas sirven como interfaces entre sus respectivas economías globales y nacionales.

Capron (2004) también recurre al amplio recorte histórico para explicar cómo en todas las sociedades se encuentran tradiciones de encerramiento e introversión del hábitat. En occidente, el deseo de protección familiar se encuentra presente en la sociedad burguesa del siglo XIX. Ella menciona que los barrios privados han existido en Londres desde el siglo XIX lo cual comprueba que no es novedad (Capron, 2004). Actualmente, en el caso de Los Ángeles, predominan las casas individuales asociadas a una sociedad fuertemente consumista, sin embargo, la segregación es una característica que se ha constatado desde el Londres victoriano y no es algo que ha surgido con el urbanismo moderno (Capron, 2004). París, por otro lado, se visualizó como un ejemplo de ciudad abierta y democrática no obstante hay que tener precaución con la escala de análisis para evitar generalizaciones fuera de lugar. Capron (2004) resalta que edificios Haussmanianos fomentaron la utilización de espacios interiores privados facilitando la homogeneización social en su interior. Este abordaje demuestra cómo la estigmatización de los barrios cerrados nos hace creer que es un resultado abominable; empero, la utilización de un amplio recorte teórico, para buscar los orígenes de los patrones residenciales, demuestra la posibilidad de otro prisma para analizar la realidad. Esto es un aporte positivo para el análisis de las mini ciudades centroamericanas desde otra perspectiva.

En cuanto a las referencias históricas centroamericanas, la comparación con la ciudad europea de antaño también es muchas veces utilizada para retomar, con nostalgia, las características físicas y funcionales de las ciudades. El autor guatemalteco Oscar Peláez Almengor (2008) publica su obra titulada El pequeño París haciendo alusión al comentario de Nevin O. Winter en Guatemala and her people of to-day (1909) el cual se refería a la importancia de Ciudad Guatemala como la más importante y grande de Centroamérica y que se le podría llamar "Little Paris". Se trata de una investigación histórica que recopila relatos de otros viajeros como Bates, H. W. (1882) en The West Indies and South America o Pepper, Charles M. (1906) en Guatemala: The Country

$2 \quad$ Concepto urbanístico introducido a finales del siglo XX que consiste en una serie de principios como, por ejemplo: los barrios deberían tener un uso y población diversa, las comunidades deberían de fomentar las áreas peatonales, los espacios urbanos deberían de ser diseñados celebrando la historia local y su cultura, el clima y la ecología del lugar; entre otras ideas (Congress For The New Urbanism, 1999). of the Future. Resulta de interés esta obra de Peláez (2008) donde se realiza una comparación con las grandes ciudades extranjeras a principios de siglo a través de la visión de los viajeros extranjeros. La importancia de estas contribuciones radica en realizar una mejor caracterización y explicación de la razón de ser de las mini ciudades como espacios o parques tematizados que emulan a la ciudad de antaño.

Para el caso costarricense, diversos autores también recurren al estudio comparativo entre San José y las ciudades europeas de antaño. Este es el caso de María del Carmen Araya (2010) con su libro San José, de "París en miniatura" al malestar en la ciudad propone una breve historia de San José como "sistema simbólico" para entender los imaginarios urbanos actuales desde el siglo XX. Ella comenta cómo 
el proceso de diferenciación geográfica en San José ha dependido de diversos personajes, instituciones o grupos que han ejercido el poder en la ciudad. Para esto ella regresa hasta el siglo XVII para comentar sobre el plan damero y aterriza en la década del 2000 para comentar sobre las polarizaciones en el Casco Urbano Central. También cabe destacar la obra de Florencia Quesada (2011) La modernización entre cafetales, San José, Costa Rica, 1880-1930 donde la autora hace referencia al proceso de europeización de las costumbres urbanas de principio del siglo XX en el periodo republicano. Al igual que para el caso guatemalteco, estas producciones contribuyen a entender detalles sobre el proceso de urbanización para, por ejemplo, explicar las actuales técnicas de marketing de las mini ciudades que aluden al pasado.

\section{III) La definición de "ciudad"}

Ciudad es una categoría de análisis que está en constante mutación. Nuevas tendencias, nuevas formas de habitación y de crecimiento urbano exigen diversas perspectivas de investigación y no, necesariamente, los proyectos tienen que encajar en las definiciones antiguas de ciudad.

Una amplia gama de autores e investigaciones buscan constantemente concluir una definición de ciudad. La tendencia suele aferrarse a las definiciones clásicas de la Escuela de Chicago y hasta de las Leyes de las Indias. Con estas definiciones poco actualizadas, las mini ciudades o proyectos de uso mixto de hoy día quedarían por fuera de la categoría de análisis de ciudad al no cumplir con las características clásicas de su definición. De esta manera, las diversas propuestas inmobiliarias que están evolucionando al pasar de los años, no encajan en las definiciones y funciones de las ciudades de la antigüedad. Otros autores tratan de ir más allá. Por ejemplo, el francés Helmer Etienne (2011) resalta que una característica importante de las ciudades en general es su dimensión política, pero esta perspectiva o definición también significaría dejar por fuera a las mini ciudades de iniciativa privada. Por otro lado, Gorelik (2005) resalta que lo que define una ciudad difícilmente sirve para otras.

Janoschka (2002) y Ford (1996) dejan clara esta necesidad de una nueva definición de ciudad latinoamericana al proponer una adaptación y actualización espacio temporal de los modelos de la Escuela de Chicago y Los Ángeles. Sus aportes toman en cuenta que las nuevas formas urbanas poseen "un carácter marcadamente insular"; detalles que no fueron incluidos en modelos tradicionales y que pueden servir para el análisis de las mini ciudades y su conectividad con la gran ciudad.

Por otra parte, el geógrafo urbano Guy Thuiller (2005) no propone una definición de ciudad como tal, pero parte del análisis de los barrios cerrados para entender el impacto que estos tienen sobre la percepción de la población sobre lo que es y debería de ser la ciudad y la vida urbana. Él se cuestiona cómo estos barrios modifican la naturaleza de la metrópolis latinoamericana y, por consiguiente yo agrego, la definición de esta misma. Este autor coincide con los aportes de Maristella Svampa (2002) la cual expone que estos espacios fragmentados de estilo "burbuja" permiten o suscitan prácticas sociales diferentes a las de los espacios públicos. Desde un punto de vista de la sociología, Svampa (2002) discute que los barrios privados, "fueron un anticipo, una muestra, una suerte de ilustración de lo que vendría". Es un anticipo de otras tipologías urbanas (como las mini ciudades) que podrían ser vistas, en palabras de la autora, como fragmentos que "contienen en sí mismos su propia lógica de acción y representación, su propio universo simbólico y cultural, sus específicos espacios de sociabilidad" en un contexto de "descomposición social" (2002).

\section{IV) Centros comerciales y parques temáticos}

En cuanto a los centros comerciales que tratan de encajar en una definición de ciudad, Etienne (2011) demuestra cómo estos tienden a emular la ciudad clásica. El autor hace una aproximación novedosa ya que normalmente las obras tratan solamente sobre barrios o solo sobre centros comerciales. Este abordaje es poco frecuente y permite entender cómo las nuevas tendencias inmobiliarias aspiran a realizar un centro urbano que contenga la función económica de la ciudad y del mercado y, también, que 
contengan símbolos tradicionales como un centro espiritual y cultural (Etienne, 2001). De la misma manera, Capron (1996) en su tesis La ville privée: les shopping centers à Buenos Aires analiza en detalle cómo los centros comerciales imitan la ciudad perfecta desde su arquitectura hasta el campo semántico utilizado en el mercadeo. El sociólogo puertorriqueño Rubén Dávila (2005) escribe El mall, del mundo al paraíso y comenta cómo los centros comerciales simulan ser ciudades con escenografías "monumentales de calles, plazas, árboles, cielo, que no son calles, plazas, árboles ni cielo". El autor se centra en la temática desde la perspectiva del "fake" y de la retórica de la mímica como una crítica a la falsificación. Siguiendo esta discusión, Etienne (2011), por su parte, resalta que los malls, que aunque traten de emular las características de una ciudad, no dejan de ser privados, concordando con el arquitecto estadounidense Michael Sorkin (1992).

El sociólogo Rodrigo Salcedo y la arquitecta Liliana de Simone presentaron el libro El Mall en Chile 30 años dónde se cuestionan "¿por qué nuestros intelectuales han dejado de cuestionar y conversar sobre el mall como se hizo en los noventa y comienzos de los dos mil?". En este contexto, los autores exponen que los malls han sido utilizados por intelectuales y políticos como "grandes metáforas de la sociedad en la que vivimos, concentrando todas sus características positivas y negativas" como una forma de criticar al modelo neoliberal. Esta producción es un excelente punto de partida para el estudio de las mini ciudades las cuales concentran características de centros comerciales.

Continuando en el contexto de la tematización, un caso ampliamente estudiado es el de las inversiones de la compañía Walt Disney en el sector inmobiliario, creando espacios residenciales semejantes a mini ciudades controladas por el sector privado. La geógrafa de la Universidad Paris 13, Sophie Didier publicó Disney urbaniste: la ville de Celebration en Floride (1997) y expone que esta ciudad reproduce la Main Street de los parques temáticos de la misma empresa. Se comenta cómo esta es una manera de hacer referencia a un sistema de valores tradicionales para intentar remediar la crisis urbana que vive el desarrollo periférico el cual huye del centro en los años 1950 en Estados Unidos, tal y como lo expuso Victor Gruen en su libro The heart of our cities (1967). Gruen es un autor que cabe destacar ya que se considera el "padre del mall". Él se dedicó al diseño de diversos centros comerciales en Estados Unidos en la época post Segunda Guerra como un antídoto a los problemas del intenso proceso de dispersión urbana. En Shopping towns USA, The planning of shopping centres (1960), el autor explica, paso a paso, cómo diseñar un centro comercial, cuáles son los actores implicados y hasta cómo son los detalles de arquitectura para asegurar un éxito comercial. Uniendo la temática del mall y del Main Street de Disney, el estadounidense William Severini Kowinski en su libro The Malling of America (1985), comenta que el mall no solo actuaba como un Main Street sino que es diseñado como tal con el objetivo de llenar los deseos de sus usuarios y eliminar sus miedos del entorno.

Sorkin escribió See you in Disneyland publicado en Variations on a Theme Park: The New American City and the End of Public Space (1992) donde también hace referencia a este concepto de ciudad idílica creada por Disney, refiriéndose a ella como altamente regulada y que provee una experiencia simplificada y limpia, versus las complejidades de la ciudad. Este autor hace analogías entre la realidad y la fantasía vendida por el parque temático y utiliza el término "disneyificación" para referirse al proceso que está experimentando la cultura. Cabe resaltar que Sorkin (1992) compara las semejanzas entre las ciudades jardín de Ebenezer con el Parque Disney ya que comparten características como su construcción en las afueras de la vieja ciudad sobrepoblada, sus cortas distancias caminables y la separación del peatón y el automóvil. A pesar de que Sorkin (1992, p. 231) critica que la "zona Disney" no es para nada "urbana" e "invoca un urbanismo sin producir una ciudad", la importancia de este abordaje radica en que la ideología de las ciudades jardín y los principios del Nuevo Urbanismo, pueden ser vistos como la base de la planificación de las nuevas mini ciudades centroamericanas.

Christine Boyer (1992), profesora de urbanismo en la Universidad de Princeton, presenta Cities for sale: Merchandising History at South Street Seaport donde ella expone que las ciudades son puestas a la venta. También da el ejemplo del Main Street de Disneyland y comenta que el American way of living es visto como un símbolo universal de progreso que puede ser consumido o comprado. La autora explica cómo la nostalgia "is a sweet 
sadness" generada por el sentimiento de que algo falta en el presente. Se refiere a la experiencia de lo auténtico, a un pasado más agradable, como si fuese un sentimiento de querer volver a poseer y experimentar algo no afectado por el tiempo, semejante a la idea expuesta por Janoschka (2005) o a los imaginarios urbanos identificados María del Carmen Araya (2010) en San José. La conexión de estas ideas con las mini ciudades es la identificación y análisis de ese anhelo por revivir un pasado que se evidencia, tanto en la arquitectura como en la toponimia y en la simbología utilizada por los promotores del mercado inmobiliario para crear espacios de inmersión.

\section{V) El carácter dinámico de las ciudades}

Otra tendencia encontrada en la literatura revisada resulta ser el énfasis del carácter cambiante de las estructuras de las ciudades. El filósofo brasileño Nelson Brissac (2009) subraya que el espacio urbano se adapta a los cambios asociados a la globalización. En el marco del estudio de las mini ciudades, sus aportes encajan de manera enriquecedora al mencionar si no deberíamos de pensar en la megaciudad latinoamericana como la vanguardia de un nuevo tipo de urbanidad. Este aporte resulta inspirador en el contexto de la identificación de las diversas novedades inmobiliarias.

Ford (2000), por su parte, enfatiza el carácter dinámico de las ciudades a lo largo del tiempo. Existen nuevas dinámicas, flujos de inversión y un cambio en la infraestructura del transporte, los cuales son algunos de los factores expuestos para visualizar los cambios urbanos que se han dado en el tiempo.

En el caso de Alladi Venkatesh (1991), recurre más a un análisis orientado a las estadísticas para demostrar los efectos de la transformación postsuburbana en los patrones de consumo en diferentes escalas. Al igual que Brissac (2009), que Ford (2000) y que Gotsch (2009), Venkatesh (1991) rescata el aporte del análisis a diversas escalas para enfatizar los cambios a nivel macro y cómo estos afectan a nivel micro. Se expone cómo las relaciones con la economía internacional tienen un efecto sobre los patrones de consumo. Por ejemplo, en el Condado Orange, demostró que se ha dado un cambio de patrón de propietarios de pequeños comercios locales hacia el surgimiento de grandes centros comerciales que venden marcas internacionales. Esto resulta interesante para analizar la fuente del capital inmobiliario envuelto en la creación de las nuevas mini ciudades.

Ford (1996), Janoshcka (2002) y Borsdorf (2003), concuerdan en presentar actualizaciones de modelos urbanos adaptados a la ciudad contemporánea en Latinoamérica. Ford (1996) enfatizó el carácter cambiante de las ciudades y sugiere, por lo tanto, que los modelos urbanos sean flexibles para adaptarse a las condiciones de cada localidad. Borsdorf (2003), por su parte, comenta la constante reorganización del espacio y el carácter dinámico de las ciudades ya que estas no solo homogenizan sino que también acentúan las particularidades locales en el contexto de la globalización. El autor sugiere que a este proceso se le llame "glocalización". Se resalta la importancia de estos aportes para, eventualmente, sugerir un debate sobre cuál sería la posición y el rol de las mini ciudades dentro de la gran ciudad Latinoamericana, por ejemplo.

\section{VI) Gaps en la producción científica: mini ciudades en Centroamérica}

No se encontraron publicaciones científicas específicamente sobre mini ciudades en Centroamérica, motivo por el cual se amplió la búsqueda al continente americano abarcando, por separado, la temática de los barrios cerrados, parques temáticos y centros comerciales. Sin embargo, esto no quiere decir que no haya aportes interesantes en estas temáticas paralelas. Se rescatan las investigaciones del Centro de Estudios Urbanos y Regionales de la Universidad de San Carlos de Guatemala, las cuales hacen un análisis local detallado de la situación local para aportar sus propias teorías sobre el proceso de metropolización. Cabe mencionar los aportes de Amanda Morán (2011) en Segregación, vulnerabilidad y exclusión social en la ciudad de Guatemala, una visión de los asentamientos precarios. Si bien es cierto no trata específicamente sobre las mini ciudades en Guatemala, sí ofrece un contexto interesante sobre la génesis de la segregación en Ciudad Guatemala desde el siglo XX. Otro investigador de este 
centro es Mario Alfonso Bravo Soto (2007) quien presentó Proceso de urbanización, segregación social, violencia urbana y "barrios cerrados" en Guatemala 1944-2002. Este expone consideraciones teóricas y metodológicas y, finalmente, aterriza en la realidad guatemalteca explicando diversos casos de barrios cerrados en la Ciudad. De esta manera, estos estudios locales sí enfatizan la existencia e importancia de las contribuciones específicas sobre el proceso de urbanización y sus tipologías. Por ejemplo, el políticólogo guatemalteco Jorge Aragón (2008) en su producción Análisis del sistema de regiones en Guatemala. La regionalización oficial y otras propuestas 19672007 se refiere a la formación del capitalismo en el contexto de la formación del territorio y su posterior regionalización para entender la división político administrativa en la actualidad. El economista y sociólogo guatemalteco Eduardo Velásquez (2016), con su reciente producción La nueva Guatemala de la Asunción: Economía política, crecimiento urbano y urbanización 1898-1954; Tomo I: 1989-1931, también realiza una detallada periodización histórica sobre el proceso de crecimiento urbano vinculado al desarrollo económico, social y político. Estas obras, aunque parecieran no estar vinculadas con el tema de las mini ciudades, permiten tener un acercamiento a la dinámica actual urbana para pensar procesos como el de fragmentación social y espacial que caracterizan el auge de estas nuevas tipologías urbanas centroamericanas.

Otras obras centroamericanas incluyen a la antropóloga social Manuela Camus que investiga, desde el Centro de Estudios de Género de la Universidad de Guadalajara, México, y presenta el artículo Mujeres y neocolonialidad en los condominios de lujo de ciudad de Guatemala (2015). Este aporte da una visión clara de la vida en los condominios suburbanos de clases medias y medias-altas que se extienden por el sureste de la ciudad de Guatemala y la tendencia de habitar en fraccionamientos cerrados, como lo podrían llegar a ser las mini ciudades.

El sociólogo Azael Carrera Hernández presenta, desde la Universidad de Panamá, Impacto de la política habitacional sobre la segregación espacial y la formación de guetos urbanos en la ciudad de Panamá en el marco de Estudios sobre políticas públicas en América Latina y el Caribe: Ciudadanía, democracia y justicia social de CLACSO (Consejo Latinoamericano de Ciencias Sociales). El comenta temáticas sobre la globalización neoliberal y las políticas de vivienda y cómo estas fomentan la generación de una cultura de la segregación en los barrios periféricos de la ciudad. Este aporte cabe considerarlo en la discusión de mini ciudades, ya que contribuye con una discusión valiosa sobre el papel del Estado en el la dinámica de integración de estas tipologías urbanas en los recursos que ofrece la ciudad.

Otro tema muy próximo a las mini ciudades resulta ser el de las Charter Cities en Honduras. Él proyecto fue iniciado por el economista Paul Romer, alrededor de 2011, y sugería un nuevo modelo el cual implicaba que un país en desarrollo otorgara un espacio para implementar una ciudad que sería administrada por un tercero. En este contexto intentó crear una división administrativa llamada zona de empleo y desarrollo económico (ZEDE), sin embargo, la idea original de Romer, ha sido modificada. Diversos aportes científicos discuten las consecuencias y hasta legitimidad de esta forma de desarrollo propuesta. Rahul Sagar presentó Are charter cities legitimate? (2016) donde se abre una discusión mencionando que las ciudades chárter tienen más chance de ser exitosas si la comunidad internacional toma el liderazgo en su creación, ya sea induciéndolo o interviniendo, sin embargo el autor comenta que resulta una paradoja ya que entre más dependan de la intervención extranjera, más se acerca, no necesariamente al colonialismo, sino al paternalismo en la forma de un fideicomiso (Sagar, 2016). Esta es una muestra de las discusiones que giran en torno a los intentos de "crear ciudades", lo cual podría ser un punto de partida para evaluar el rol y alcances de las mini ciudades centroamericanas.

Para el caso costarricense, tampoco se encontraron producciones científicas específicamente sobre mini ciudades. En este contexto se rescatan producciones que tratan las temáticas paralelas, como es el caso de la costarricense María del Carmen Araya (2010), quien publica sobre los imaginarios urbanos de San José, Costa Rica, en el período 2000-2006. La autora aborda diversas temáticas, incluyendo la polarización social, la expansión de la lógica del consumo y el deseo de vuelta a un "centro repoblado y regenerado", entre otros temas específicos de la ciudad costarricense. El desarrollo 
de nuevos polos territoriales de desarrollo también es analizado específicamente en la ciudad San José, lo cual resulta un aporte novedoso y actualizado para visualizar el rol de las mini ciudades en este escenario.

Alejandro Alvarado y Gustavo Jiménez (2015) presentaron su tesis Urbanizaciones cerradas en Costa Rica: transformaciones socio-espaciales en la urbanidad y segregación socio-residencial en el distrito de San Rafael de Escazú (1990-2015). Ellos se interrogaron sobre las transformaciones socio-espaciales que las urbanizaciones cerradas generaron y las implicaciones en la producción de cambios en la urbanidad. Los autores comentaron que el desarrollo de las urbanizaciones cerradas se trata de un fenómeno central en los procesos de re-estructuración urbana durante las últimas dos décadas y que esto no ha sido estudiado en Costa Rica. Ellos proponen que el proceso de segregación residencial se da en dos niveles, un nivel físico y un nivel simbólico, lo cual podría ser una metodología de análisis interesante para aplicarlo a las mini ciudades en cuestión.

En el caso de documentos que sí mencionan las mini ciudades centroamericanas, son artículos de periódicos y revistas inmobiliarias señalando diversos casos costarricenses y otros, menos numerosos, de otros países centroamericanos. Artículos periodísticos han reportado diversos proyectos de uso mixto, principalmente en Costa Rica donde han sido identificados como mini ciudades (periódico La Nación, periódico Actualidades Inmobiliarias, Revista Summa, Revista Inversión Inmobiliaria, entre otros). Sin embargo, estos no entran en la categoría de aportes científicos para el presente ensayo.

\section{Análisis global de la literatura revisada}

A manera de análisis global de la literatura, el ensayo pretendió revisar críticamente las tendencias en una selección de trabajos realizados para señalar cuáles son los conflictos, los patrones temáticos, las faltantes en las discusiones y las tendencias de investigación, para así poder aportar un contexto adaptado a la realidad centroamericana, sin caer en la generalización y así encauzar nuevos estudios sobre una temática tan dinámica y diversa. Los aportes científicos sobre las tipologías urbanas mencionadas son vastos y no se pretendió redundar en discusiones ya planteadas.

Las mini ciudades son una tendencia que ha caracterizado el reciente auge inmobiliario en la segunda década del siglo XXI y no se registró ningún aporte científico sobre el tema específico. La temática resulta ser tan amplia, que sugiero un abordaje que tome en cuenta las diversas tipologías planteadas para analizar estos proyectos híbridos.

Capron (1996; 2004) y Gotsch (2009) realizaron detallados estudios de proyectos lo más semejantes a las mini ciudades centroamericanas pero con casos de estudio europeos, asiáticos o brasileños y solo utilizando algunas de las categorías propuestas, no todas simultáneamente. Las discusiones de las Charter Cities de Paul Romer también se aproximan lo suficiente al caso de las mini ciudades como un punto de partida y de comparación.

A pesar de que la temática investigada y las categorías propuestas ya han sido individualmente estudiadas desde hace décadas, cabe rescatar como metodología, que muchos temas clásicos como el de las ciudades jardín o ciudades coloniales sirven de base para poder entender el direccionamiento de las tendencias actuales.

Se observó que el carácter dinámico del crecimiento urbano y la especificidad del lugar subrayan que cada país, región y continente, experimentan experiencias a diversos ritmos y direcciones que merecen un estudio específico de su realidad. Nuevas definiciones y parámetros están constantemente surgiendo y, así, se justifica que es imprescindible analizar las dinámicas de los nuevos proyectos que se asemejan a mini ciudades.

La mayoría de los aportes científicos están enfocados en algunos países y no deberían de representar una realidad generalizada. No todos los países latinoamericanos tienen tanta riqueza literaria en la geografía urbana como Estados Unidos, Brasil o Argentina, por ejemplo. La región centroamericana cuenta con menos aportes y estudios en el área de la geografía urbana y estos se centran principalmente en el tema de los 
barrios cerrados y la segregación urbana. Agrego que, con este resultado, es necesario establecer nuevas formas de observación para los múltiples procesos y agentes envueltos. En este contexto sugiero seguir las recomendaciones de Gotsch (2009) para iniciar un estudio actualizado de la situación actual centroamericana en cuanto a las mini ciudades o NeoTowns y analizar si ambas categorías se podrían tratar bajo la misma tipología.

Se perciben muchos gaps en la cantidad y la temática expuesta entre países. Muchas veces Latinoamérica se toma en cuenta como si fuera un solo "laboratorio" donde todos los casos de estudio deberían de encajar en las mismas conclusiones. Diversos autores resaltaron esta problemática y pocos enfatizan la importancia de los aportes de los estudios comparativos.

Se concluye reflexionando sobre la necesidad de aportar un análisis localizado y actualizado sobre las mini ciudades como recientes formas de metropolización y nuevas tipologías urbanas en Centroamérica, subrayando la importancia de seguir un abordaje que incluya diversas escalas espacio-temporales en las diferentes categorías de análisis desde la perspectiva de la geografía urbana. Así mismo, se sugieren estudios comparativos para ampliar el espectro de análisis entre las diferentes tipologías de diversos países para contextualizar, mejor aún, las dinámicas urbanas indagadas.

\section{Referencias bibliográficas}

Alvarado, A y Jiménez, G. (2015). Urbanizaciones cerradas en Costa Rica: transformaciones socio-espaciales en la urbanidad y segregación socioresidencial en el distrito de San Rafael de Escazú (1990-2015). XXX Congreso Latinoamericano de Sociología. Costa Rica, 2015.

Aragón, J. (2008). Análisis del sistema de regiones en Guatemala. La regionalización oficial y otras propuestas. 1967-2007. Serie Territorio y Regionalización en Guatemala. Volumen I. Guatemala: Centro de Estudios Urbanos y Regionales.

Araya. M. (2010). San José, de "París en miniatura" al malestar en la ciudad. San José: EUNED.

Bates, H. W. (1882). The West Indies and South America. London: Edward Stanford.

Blackely, E. J. y Snyder, M. G. (1997). Fortress America, Gated Communities in the United States. Massachusetts: The Brookings Institution.

Borsdorf, A. (2003). Hacia la ciudad fragmentada. Tempranas estructuras segregadas en la ciudad latinoamericana. Scripta Nova. Revista electrónica de geografía y ciencias sociales, VII (146)(122). Barcelona: Universidad de Barcelona. Recuperado de http://www.ub.es/geocrit/sn/sn-146(122).htm

Borsdorf, A. (2003). Cómo modelar el desarrollo y la dinámica de la ciudad latinoamericana. EURE (Santiago), (29)86, 37-49. Recuperado de http://www. scielo.cl/scielo.php?script=sci_arttext\&pid=S0250-71612003008600002\&lng=es \&nrm=iso

Boyer, M. C. (1992). Cities for sale. En SORKIN, M. (Ed.), Variations on a Theme Park: The New American City and the End of Public Space (pp. 181-204). New York: Hill and Wang.

Bravo, M. A. (2007). Proceso de urbanización, segregación social, violencia urbana y "barrios cerrados" en Guatemala 1944-2002. En: CEUR. Proyecto Macro: El proceso de urbanización en Guatemala 1944-2002. Volumen VI. Inédito. Guatemala: Centro de Estudios Urbanos y Regionales.

Brissac, N. (2009). Latin American Megacities: the new urban formlessness. En Biron, R. City/Art The urban scene in Latin America (pp. 233-265). Durham and London: Duke University Press.

Camus, M. (2015). Mujeres y neocolonialidad en los condominios de lujo de ciudad de Guatemala. Anuario de Estudios Centroamericanos, 4: 191-218. 
Capron, G. (2004). Les ensembles résidentiels sécurisés dans les Amériques: une lecture critique de la littérature. L'espace géographique, 2 (33), 97-113.

Capron, G. (1996). La ville privée: les shopping centers à Buenos Aires. (Tésis de doctorado en Geografía-Ordenamiento). Université Toulouse II. Toulouse.

Campbell, R. (1981). Evaluation: Boston's upper urbanity. Faneuil Marketplace after five years. A/A Journal, 6: 25-32.

Congress for the New Urbanism. (1999). Charter Of The New Urbanism. Nueva York: McGraw-Hill.

Dávila, R. (2005). El mall, Del mundo al paraíso. San Juan: Ediciones Callejón.

Didier, S. (1999). Disney urbaniste: la ville de Celebration en Floride. Cybergeo: European Journal of Geography. doi: 10.4000/cybergeo.1147

Etienne, H. (2011). La ciudad contemporánea, ¿una polis sin política? En Boletín Científico Sapiens Research, 1 (2), 88-91.

Ford, L. (2000). Cities and Urban Land Use in Advanced Placement Human Geography. Journal of Geography, 99(3): 153-168. doi:10.1080/00221340008978910

Ford, L. (1996). A new and improved model of Latin American city structure. The Geographical Review, 86 (3), 437-440.

Fuller, B., Romer, P. (2012). Success and the City: How Charter Cities Could Transform the Developing World. Macdonald-Laurier Institute. Recuperado de http://www. macdonaldlaurier.ca/files/pdf/How-charter-cities-could-transform-the-developingworld-April-2012.pdf

García, B. y Pérez, T. (2004). Hacia la ciudad amurallada. International Planning History of Urbanism. 1-16. Recuperado de http://www.etsav.upc.es/personals/iphs2004/ pdf/067_p.pdf

Gorelik, A. (2005). Produção da "cidade latino-americana. (traducción de Fernanda Arêas Peixoto). Tempo Soc, 17(1). Recuperado de http://www.scielo.br/scielo. php?script=sci_arttext\&pid=S0103-20702005000100005

Gotsch, P. (2009). NeoTowns, Prototypes of corporate urbanism. (Tésis en ingeniería). Universidad de Karlsruhe, Alemania.

Gruen, V, y Smith, L. (1960). Shopping towns USA. The planning of shopping centers. New York: Reinhold Publishing Corporation.

Gruen V. (1967). The Heart of our cities. The urban crisis: diagnosis and cure. New York: Simon and Schuster.

Hardwick, J. M. (2003). Mall Maker: Victor Gruen, Architect of an American Dream. Pensilvania: University of Pennsylvania Press.

Janoschka, M. (2002). El nuevo modelo de la ciudad latinoamericana: fragmentación y privatización. Revista Eure, XXVIII (85), 11-29

Janoschka, M. (2003). Nordelta - ciudad cerrada. El análisis de un nuevo estilo de vida en el gran Buenos Aires. Scripta Nova., VII(146)(121), 1-12. Barcelona: Universidad de Barcelona. Recuperado de http://www.ub.es/geocrit/sn/sn-146(121).htm

Janoschka,, M. (2005). Discursos de inseguridad y la ciudad cerrada: mitos, realidades, barreras y fronteras de un producto inmobiliario "perfecto". Revista Imaginales. Julio-Diciembre, 11-36.

Janoschka,, M. (2011). Geografías urbanas en la era del neoliberalismo. Una conceptualización de la resistencia local a través de la participación y la ciudadanía urbana. Investigaciones Geográficas (Mx). Recuperado de http://www.redalyc. org/articulo.oa?id=56921271009

Janoschka, M. y Borsdorf, A. (2004). Condomínios fechados and Barrios privados: the rise of private residencial neighbourhoods in Latin America. En Glasze, G., Webster, C. y Frantz, K. (Ed.): Private Neighbourhoods. Global and local perspectives. London: Routledge, in press. Recuperado de https://www.uibk.ac.at/geographie/ personal/borsdorf/pdfs/buch_gc_-_janoschka-borsdorf_abgabeversion.pdf 
Kling, R., Olin, S., y Poster, M. (Eds.) (1991). Postsuburban California: the transformation of Orange County since World War II. Berkeley: University of California Press.

Kowinski, William Severini (1985). The Malling of America. New York: William Morrow and Company Inc.

Lins, E. (2009). Urbanizaciones temáticas: arquitecturas del deseo. Revista Urban perspectives/Perspectivas urbanas. 10, 13-21. Recuperado de http://www.etsav. upc.es/personals/iphs2004/urbper/num10/index.htm

Macleod, G. y Ward, K. (2002). Spaces of Utopia and Dystopia: Landscaping the Contemporary City. Geografiska Annaler: Series B, Human Geography, 84, 153170. doi: 10.1111/j.0435-3684.2002.00121.x

Méndez, E. (2004). Vecindarios defensivos latinoamericanos, los espacios prohibitorios de la globalización. Revista Urban perspectives / Perspectivas urbanas. 4, 2004, 1-17. Recuperado de http://www.etsav.upc.es/personals/iphs2004/urbper/num04/ index.htm

Morán Mérida, A. (2011). Segregación, vulnerabilidad y exclusión social en la ciudad de Guatemala, una visión de los asentamientos precarios. Universidad de San Carlos de Guatemala, Guatemala: Centro de Estudios Urbanos y Regionales.

Murray, M. J. (2004). The Evolving Spatial Form of Cities in a globalising world economy, Johannesburg and São Paulo. Cape Town: HSRC Publishers.

Quesada, F. (2011). La modernización entre cafetales, San José, Costa Rica, 18801930. San José: Editorial UCR.

Peláez, O. El pequeño París. (2008). Guatemala: CEUR.

Pepper, C. M. (1906). Guatemala, The country of the Future. Washington D.C.

Sagar, R. (2016), Are Charter Cities Legitimate?. Journal of Political Philosophy, 24: 509-529. doi:10.1111/jopp.12089

Salcedo, R. y de Simone, L. (2012). El Mall en Chile 30 años. Santiago de Chile: Uqbar Editores.

Soja, E. (1992). Inside Exopolis: Scenes from Orange County. En SORKIN, M. (Ed.). Variations on a Theme Park: The New American City and the End of Public Space (pp. 94-122). New York: Hill and Wang.

Sorkin, M. (Ed.) (1992). Variations on a Theme Park: The New American City and the End of Public Space. New York: Hill and Wang.

Svampa, M. (2002). Los riesgos impensados del paraíso. Revista Enfoques. Buenos Aires, 2002. Recuperado de http://www.maristellasvampa.net/archivos/period01. pdf

Thuiller, G. (2005). El impacto socio-espacial de las urbanizaciones cerradas: el caso de la Región Metropolitana de Buenos Aires. Revista Eure. XXXI(93): 5-20.

Velásquez C., E. (2016). La nueva Guatemala de la Asunción: Economía política, crecimiento urbano y urbanización 1898-1954. Tomo I: 1989-1931. Guatemala: Centro de Estudios Urbanos y Regionales.

Winter, N.O. (1909). Guatemala and her people of to-day. Boston: L.C. Page and Company. 